\title{
Comportamiento organizacional ciudadano (COC): Una propuesta para valorar la convivencia escolar desde las conductas de ciudadanía
}

\section{Citizen Organizational Behavior (COC): A proposal to assess school coexistence from citizenship behaviors}

DOI: http://dx.doi.org/10.17981/cultedusoc.8.2.2017.03

Irmina Hernandez-Sánchez ${ }^{1}$

\begin{abstract}
Resumen
El objetivo de este ensayo fue ampliar la comprensión acerca de la convivencia escolar, haciendo una breve reflexión acerca de lo que significan las conductas de ciudadanía en las instituciones educativas y como estos comportamientos podrían abordarse desde el modelo del comportamiento organizacional ciudadano (COC) desarrollado por Spector (2002). Para alcanzar este objetivo, se planteó el tema de la convivencia escolar a partir de la propuesta del Ministerio de Educación Nacional y algunos otros autores; así como se describió que se entiende por conductas de ciudadanía y finalmente se muestra los elementos generales que componen el modelo de COC y su utilidad frente al tema de la convivencia escolar.
\end{abstract}

Palabras clave: Convivencia escolar, conductas de ciudadanía y comportamiento organizacional ciudadano.

1 Doctorante en formación, Magister en Psicología, Psicóloga, Técnica superior universitaria en Educación Especial, Docente e investigadora Universidad de la Costa. (CUC), Barranquilla, Colombia. Correo: ihernand8@cuc.edu.co 


\begin{abstract}
The objective of this essay was to expand the understanding of school coexistence, making a brief reflection about what citizenship behaviors mean in educational institutions and how these behaviors could be addressed from the model of citizen organizational behavior (COC) developed by Spector (2002). To achieve this goal, the topic of school coexistence was raised based on the proposal of the Ministry of National Education and some other authors; as well as it was described that citizenship behaviors are understood and finally the general elements that make up the COC model and its usefulness in relation to the topic of school coexistence are shown.
\end{abstract}

Key words: School coexistence, citizenship behavior and citizen organizational behavior.

\title{
Introducción
}

\section{Convivencia escolar}

La convivencia escolar es un tema de gran interés dentro del ambiente educativo; autores como Fierro y Caso (2013) señalan que este fenómeno y su evaluación implican el foco de estudio de un conjunto amplio de investigadores quienes, quienes buscan contribuir a la comprensión y a la mejora de la calidad de la vida en los centros educativos.

De acuerdo al informe Delors (1997), el constructo de convivencia escolar representa un pilar fundamental para la educación. Aprender a convivir, no es meramente un aspecto derivado del logro académico ni una estrategia para prevenir y/o evitar la violencia en los centros educativos; tiene que ver más con un aspecto fundamental para la vida.

Para Fierro y Caso (2013) el núcleo de la discusión alrededor de la convivencia se enmarca en la siguiente interrogante: ¿la convivencia se trata de una estrategia de carácter remedial para prevenir la violencia en las escuelas, apuntar al logro educativo y/o atender el fracaso y la exclusión en la escuela o tiene importancia por sí misma?

El Ministerio de Educación Nacional en Colombia, considera a la convivencia escolar como un elemento fundamental para una educación con calidad; entendiendo ésta como: aquella que forma ciudadanas y ciudadanos con valores éticos, respetuosos de lo público, que ejercen los derechos humanos, cumplen sus deberes sociales y conviven en paz... (MEN 2013, p. 5)

Al respecto, Carbajal-Padilla (2013) hace la salvedad de que la calidad de la educación no sólo implica el desarrollo de conocimientos y habilidades cognitivas, sino que además incluye el desarrollo de habilidades para una ciudadanía responsable dentro de ambientes emocionalmente enriquecedores.

En este sentido ; es así que en el 2013 se promulga la ley 1620, mediante la cual, el Congreso de Colombia crea mecanismos de prevención, protección, detección temprana y de denuncia ante las autoridades competentes, de todas aquellas conductas que 
atenten contra la convivencia escolar y la ciudadanía entre otros aspectos (Congreso de Colombia , 2013)

A partir de lo dicho, es pertinente establecer que se entiende por convivencia escolar y que elementos la conforman, para así poder comprender su relevancia en el entorno educativo.

López de Mesa-Melo, Carvajal-Castillo, Soto-Godo y Urrea-Roa, (2013) expresan que la convivencia es una manera de socializar con el otro en su cotidianidad y con la cual debe llegar a cohabitar compartiendo criterios de acuerdo con su proceso de desarrollo. Así mismo, los autores señalan que convivir hace parte del reconocimiento de que quienes comparten por diversos motivos escenarios y actividades dentro de un sistema convencional, están sujetos a normas que tienen el fin de favorecer la interacción, prevenir los conflictos y sus consecuencias (López de Mesa-Melo et al 2013, p. 387)

Carbajal-Padilla (2013), al referirse a la convivencia propone dos visiones de este constructo; la primera postura hace referencia a un concepto restringido de la convivencia, el cual se centra en torno a la disminución de los niveles de violencia escolar, haciendo énfasis en el control de los comportamientos agresivos de los alumnos.

La segunda visión descrita por la autora, implica una perspectiva de la convivencia escolar mucho más amplia, integrando las relaciones democráticas (institucionales, culturales e interpersonales) y las estructuras de participación como elementos esenciales para la construcción y consolidación de la paz (Carbajal-Padilla, 2013).

Esta segunda perspectiva, a diferencia de concentrarse en controlar el comportamiento agresivo de los estudiantes (paz negativa), busca la transformación de las prácticas en el aula y en la escuela con el objeto de construir comunidades justas, incluyentes y democráticas (paz positiva). Desde esta perspectiva, la convivencia escolar permea todo el currículum educativo (Carbajal-Padilla, 2013).

De acuerdo a la autora, esta noción de convivencia escolar democrática parte de dos posturas fundamentales:

La idea de Dewey (1996) de construir en las escuelas comunidades democráticas que promuevan el crecimiento humano y las habilidades ciudadanas, y el concepto de Freire (1970) de praxis como una acción de reflexión educativa que finalmente culminará en la erradicación de la opresión y en la construcción de relaciones estructurales más equitativas. Las teorías educativas de Dewey y Freire tienen principalmente dos aspectos en común: por una parte el fomento de la comunicación abierta a través del diálogo respetuoso, y por otra la deliberación crítica y la acción dirigidas a la transformación de los obstáculos que se interponen en la realización personal. En otras palabras, Dewey y Freire convergen en el desarrollo de una educación para la ciudadanía crítica (Carbajal-Padilla 2013, p. 19).

En función de las posturas descritas, pareciera que la convivencia escolar, más allá de convivir con el otro, implica una construcción de espacios que sean incluyentes, y en donde se respire una comunidad educativa orientada a la participación y el ejercicio de la ciudadanía.

Partiendo de esta idea, luce relevante señalar como desde la ley 1620, se establece las competencias ciudadanas, como un componente fundamental para el desarrollo de esa convivencia escolar. 


\section{Competencias ciudadanas}

Se entiende por competencias ciudadanas: el conjunto de conocimientos y de habilidades cognitivas, emocionales y comunicativas que, articulados entre sí, hacen posible que el ciudadano actúe de manera constructiva en una sociedad democrática (Ley 1620).

De acuerdo al Ministerio de Educación Nacional, mediante el desarrollo de estas competencias se pretende desarrollar en los niños herramientas necesarias para relacionarse con otros, desde la comprensión y la justicia; de tal manera que sean capaces de resolver problemas cotidianos (MEN, 2004.)

Estas competencias facilitan la contribución a la convivencia pacífica, a la participación responsable y constructiva en los procesos democráticos, y al respeto a la pluralidad y la diferencia (MEN, 2004).

Partiendo de estas ideas, podría esperarse que el modelo del comportamiento organizacional ciudadano (COC), expuesto por Spector (2002), sea una herramienta para la valoración de las competencias ciudadanas y por ende de la convivencia escolar; entendiendo que las escuelas representan organizaciones orientadas al servicio educativo.

El comportamiento organizacional ciudadano o conductas de ciudadanía en las organizaciones, han sido descritas como comportamientos pro sociales, voluntarios que emiten los empleados dentro de la empresa; cuyo propósito fundamental es apoyar y ayudar a los compañeros de trabajo, así como a la organización en general, mejorando el entorno laboral afectando positivamente el clima organizacional. (Spector, 2002)

Desde principio de los años 60 autores como por ejemplo Katz y Kaht, han señalado que el funcionamiento óptimo de una organización depende, entre otras variables, de conductas que van más allá del rol que el individuo cumple dentro de dicha organización (citado en. Bateman y Organ 1983).

Estos comportamientos, de acuerdo a Bateman y Organ (1983) no se encuentran en la descripción de cargo que ocupa el empleado, más bien tienen que ver con comportamientos pro-sociales entre los que se destacan los siguientes:

- Ayuda a compañeros en problemas relacionados con el trabajo.

- Aceptación de órdenes sin mayor preocupación.

- Tolerancia ante situaciones temporales.

- Ayuda en mantener limpia y ordenada el área de trabajo.

- Promoción de un clima de trabajo agradable minimizando aquellas distracciones creadas por conflictos interpersonales.

Podsakoff, MacKeinzie, Paine, y Bachrach (2000), señalan que las conductas discrecionales son comportamientos que no son obligatorio del rol del empleado, sino que son una elección personal, por lo que su omisión no es castigada por la organización.

Para Schnake y Dumler (2003) estas conductas pro sociales, van dirigidas tanto a ayudar a un individuo en particular, como a un grupo. 
Organ (1988) plantea que estas conductas pro sociales pueden dividirse en cinco dimensiones fundamentales a saber:

a. Altruismo: trabajo voluntario para ayudar con una tarea o problema en la organización.

b. Cortesía: ser atento y respetuoso con los derechos de las demás personas.

c. Rectitud: ser puntual, tener una asistencia mejor que la norma del grupo, y seguir sensatamente las reglas normas y procedimientos de la empresa.

d. Deportivismo: se refiere a evitar quejas, agravios menores, chismes y problemas falsamente magnificados.

e. Virtud cívica: participación responsable en la vida política de la organización.

Arredondo-Trapero, Ferrer y Villa-Castaño (2011) al hacer referencia a estas conductas enfatizan que, mejoran el entorno laboral, mediante la participación voluntaria, promoviendo acciones efectivas dentro de la organización.

\section{Conclusión}

El adaptar la visión del comportamiento organizacional ciudadano (COC), al ámbito escolar reflejaría elementos relacionados con la convivencia como por ejemplo las relaciones de ayuda y apoyo al compañero, el respeto al otro, el seguimiento de la normatividad, la participación y el manejo de las situaciones conflictivas relacionadas con agravios menores, comentarios etc.

Así mismo, la valoración de estas conductas de ciudadanía podría abarcar a la comunidad educativa en general, incluyendo estudiantes, maestros y directiva.

Además el estudio desde esta perspectiva podría abrir espacios de líneas de investigación que relacionen convivencia escolar, conductas de ciudadanía en la organización y clima social escolar, de tal manera de contribuir al valor teórico del conocimiento científico, además de aportar elementos que permitirían extraer aspectos que orienten la intervención para la mejora de la convivencia escolar.

\section{Referencias}

Arredondo-Trapero, R., Ferrer, J. A. \& Villa-Castaño, L. (2011). Comportamiento ciudadano organizacional y RSE. Cuadernos de Administración, 24, (43), 221-239.

Bateman, T. \& Organ, D. (1983). Job satisfaction and the good soldier: the relationship between affect and employee citizenship. Academy of Management Journal, $26,(4), 587-595$.

Carbajal-Padilla, P. (2013). Convivencia democrática en las escuelas. Apuntes para una conceptualización. Revista Iberoamericana de Evaluación Educativa. 6, (2), 13-36. Recuperado de http://www.rinace.net/riee/numeros/vol6-num2.html

Congreso de Colombia (2013). Ley $\mathrm{N}^{\circ} 1620$.

Delors, J. et al (1998). La Educación encierra un tesoro. París, Francia: UNESCO 
Fierro, M. \& Caso, J. (2013). Sección Temática: Evaluación y análisis de prácticas de intercambio y convivencia escolar. Revista Iberoamericana de Evaluación Educativa. 6, (2), 7-12. Recuperado de http://www.rinace.net/riee/numeros/vol6-num2.html

López de Mesa-Melo, C; Soto-Godoy, M; Carvajal-Castillo, C. \& Nel UrreaRoa, P. (2013). Factores asociados a la convivencia escolar en adolescentes Educación y Educadores, 16, (3), 383-410

Ministerio de Educación Nacional (2004). Serie Guía No 6: Estándares Básicos de Competencia Ciudadana. Bogotá, Colombia: Ministerio de Educación Nacional

Ministerio de Educación Nacional (2013). Guías pedagógicas para la convivencia escolar Ley 1620 de 2013 - Decreto 1965 de 2013. Bogotá, Colombia: Ministerio de Educación Nacional.

Organ, D. (1988). Organizational citizanship behavior: the good soldier syndrome. Lexington: Lexington Books

Podsakoff, P., MacKeinzie, S., Paine, J. \& Bachrach, D. (2000). Organizational citizanship behavior: a critical review of the theoretical and empirical literature and suggestions for future research. Journal of Management, 26, (3), 513-563.

Schnake, M. \& Dumler, M. (2003). Levels of measurement and analysis issues in organizational citizenship behavior research. Journal of occupational and organizational psychology, 76, 283-30.

Spector, P. (2002). Psicología industrial y organizacional: investigación y práctica. México: El Manual Moderno. 